Tequio 1(1) 2017: 14-19

ISSN: 2594-0546

\title{
POLÍTICA EDUCATIVA Y PROFESIONALIZACIÓN DOCENTE EN EDUCACIÓN BÁSICA
}

\author{
Claudio Escobar-Cruz ${ }^{1}$ \\ Wenceslao Sergio Jardón-Hernández ${ }^{2}$ \\ Margarita Berenice Gutiérrez-Hernández ${ }^{3}$ \\ Fecha de recepción: 4 de noviembre de 2016 \\ Fecha de aceptación: 25 de mayo de 2017
}

Resumen - La reforma educativa es la expresión de una política que impulsa, al menos en el discurso, la formación integral de los alumnos de educación básica. La reforma educativa implementada en los últimos años en México se caracteriza por un conjunto de metas comunes: lograr mayor equidad en el acceso a la educación, mejorar la calidad del servicio, informar de los resultados de la educación y revisar los contenidos que se integran al currículo. A la par, se señala una serie de problemas, como altos índices de repetición, el menor dominio de aprendizajes por parte de los estudiantes o falta de renovación de los métodos de enseñanza, para lo cual se instituyó de manera "obligatoria" la propuesta de profesionalizar a los maestros como mecanismo que coadyuve a solucionar la problemática educativa. Bajo esa lógica, elevar la calidad de la educación implica mejorar el desempeño de los docentes.

\section{Palabras clave:}

Calidad de la educación, evaluación, política educativa, profesionalización docente, reforma educativa.
Abstract - The education reform is an expression of on educational politics that drives, at least in speech, the integral formation of student in basic education. The characteristic of the education reforms implemented in recent years in Mexico, identify themselves by a set of common goals: achieving greater equity in access to education, improve service quality, report the results of education, review the contents that are integrated into the curriculum. So a series of problems linked identify with the "lack of quality education "so as: high repetition rates, the best domain of learning by students, lack of renewal of teaching methods. That is why this proposal was made to professionalize teachers as a mechanism that contributes to the solution of the educational problems.

For the purposes of the current reform, raise the quality of education implies improving the performance of teachers and student, involve parents give schools de support material and review the plan and curriculum. To achieve from the official optical is essential to strengthen the valuation processes the progress and opportunities to have an education increasingly higher quality.

\section{Keywords:}

Education reform, education politics, quality of education, professionalization, evaluation.

\footnotetext{
${ }^{1}$ Candidato a maestro en Sociología. Profesor de Tiempo Completo. Universidad Pedagógica Nacional, Unidad 095 Azcapotzalco. Cuerpo académico Planeación, Política y Diversidad. Correo electrónico: vangogh7610@gmail.com

2 Doctor en Ciencias Sociales. Profesor de Tiempo Completo. Universidad Pedagógica Nacional, Unidad 095 Azcapotzalco. Cuerpo académico Planeación, Política y Diversidad. Correo electrónico: 49jardon@gmail.com

${ }^{3}$ Doctora en Gerencia y Política Educativa. Profesora de Tiempo Completo. Universidad Pedagógica Nacional, Unidad 095 Azcapotzalco. Cuerpo académico Planeación, Política y Diversidad. Correo electrónico: berenicegutierres773@hotmail.com
} 


\section{Presentación}

nte paisajes tan poco alentadores que se viven en México, plagados de violencia, corrupción, inseguridad, desempleo, precarización laboral, entre otros, es importante revisar el papel de la educación, el desempeño de los diferentes actores como la escuela, los estudiantes, padres de familia y, principalmente en el caso que nos ocupa, el docente, cuyo ejercicio resulta fundamental respecto de la dinámica que se desarrolla en espacios como la escuela y el aula, donde se lleva a cabo el quehacer de educativo. Ningún aspecto que se refiera a la educación puede dejar de ser relevante, pero consideramos clave en todo esto la acción que realiza el profesor, de ahí nuestro interés por buscar y reconocer las principales problemáticas que enfrenta la subjetividad de su ser y quehacer docente; sin duda, esta profesión pocas veces ha enfrentado tantos cambios como los ocurridos en los últimos años, ya sean sociales o culturales, que impactan a los sistemas educativos. De manera puntual, se pueden identificar los requerimientos de la sociedad, que impone una formación cada vez más extensa, aunados al requisito de profesionalizar su labor, tanto por la acelerada producción de saberes que deben ser transmitidos a las nuevas generaciones, como por las exigencias de la organización de la vida social y del ejercicio de la ciudadanía. Así, la profesionalización es la nueva coordenada en la que se plantea el trabajo docente, ése es el desafío que la institución escolar y que los profesores tienen ante sí respecto de las recientes reformas educativas aplicadas en México.

\section{Planteamiento del problema}

El papel que cumple la escuela está determinado en buena medida por los momentos en que se sitúe; así, tiende a ser conservadora o reproductora cuando la sociedad es estable, estática; y progresista o transformadora cuando la sociedad es cambiante y dinámica. En los grupos sociales predominantemente estáticos, la escuela no es ni una cosa ni la otra, pues simplemente no es, ya que (casi) nadie tiene necesidad de ella.

En las sociedades que cambian y, además, saben o creen saber en qué dirección lo hacen, la escuela se convierte en un potente (manipulado y controlado) instrumento de transformación. En ese sentido, en las sociedades que cambian, pero lo hacen de manera errática o simplemente imprevisible, la escuela se ve inmersa en un desconcierto que deriva fácilmente en una crisis que supone tanto la insostenibilidad de la dinámica previa como el despliegue de nuevas oportunidades (Fernández, 200, p. 14).

Por lo anterior, las distinciones y categorías que subyacen a las prácticas educativas no son términos neutrales y descriptivos; la amalgama de acciones y discursos se interpreta como un tipo particular de conocimientos históricamente elaborados, en el que se inscriben ciertas formas de actuar, sentir, hablar y ver el mundo (Popkewitz, 1995).

Con base en lo anterior, el proyecto busca identificar los elementos que componen la política educativa en tanto mecanismo estatal que trastoca el ámbito de la identidad, la formación, actualización y profesionalización docente, además de las condiciones laborales y la normatividad del ejercicio magisterial, donde la instrumentalización de la reforma educativa confronta las tradiciones del oficio de ser docente y la autorrepresentación de sí mismo, en un entorno caracterizado por cambios socioculturales acelerados, que a su vez presionan al propio sistema educativo para que responda a la dinamización de los

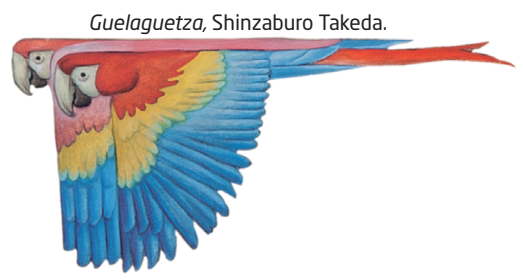

Tequio, septiembre-diciembre 2017, vol. 1, no. 1 
procesos productivos, a la veloz transformación del mercado laboral y a la mutación de la misma vida social.

\section{Hipótesis}

La política educativa, a través de la reforma educativa, impone a los docentes mecanismos de evaluación, sea para el ingreso, permanencia o promoción de una plaza, lo que implica la actualización y formación permanente, que conduce a la profesionalización del magisterio, sin que ello represente una condición automática de mejoramiento del desempeño del profesorado, ni el logro de la ansiada calidad educativa en el nivel básico.

\section{Objetivo general}

Identificar los elementos político-normativos que intervienen u obstaculizan el desempeño docente.

\section{Metodología}

Para lograr un acercamiento a la complejidad del trabajo docente, se aplicaron cuestionarios a profesoras de educación básica, inscritas y egresadas del programa de Maestría de Educación Básica que imparte la Universidad Pedagógica Nacional en dos de sus unidades, la 095 Azcapotzalco y la 098 Oriente de la Ciudad de México. A la par, se realizaron entrevistas a profundidad que arrojaron información cualitativa en relación con la manera en que se autodefinen en su condición de ser docentes, en un contexto de reforma educativa.

\section{Marco teórico: docentes, política educativa y educación básica}

Las problemáticas que enfrentan los docentes no son recientes; al menos desde la reforma educativa de 1992 se propusieron acciones para moldear a un profesional que pudiera atender las necesidades del alumno del siglo XXI, sin embargo, el esquema de actualización que hasta el momento se tiene no deja de ser tradicional. Toda la oferta sigue vertiéndose verticalmente, lo que la torna poco atractiva, además de no responder a necesidades propias del ejercicio pedagógico, ni ofrecer didácticas que los docentes requieren, por lo que, en esencia, la reforma y su propuesta de cambio se convierten en ejercicios inútiles y costosos para el gobierno, pues éste no comprende los procesos de formación ni considera los elementos involucrados para lograr una verdadera transformación educativa (Flores, 2004).

El aspecto distintivo de la política pública es el hecho de que integra un conjunto de acciones estructuradas, estables y sistemáticas en su operación, que constituyen el patrón de comportamiento o el modo como el gobierno aborda de manera permanente el cumplimiento de las funciones públicas o la atención de determinados problemas públicos. De acuerdo con el planteamiento anterior, tenemos que hacer mención de lo que se entiende por política educativa, ya que implica hablar de un conjunto de variables, de dimensiones complejas y diversas, que actúan en distintos niveles (Aguilar, 2008; Rivas, 2004).

Compartimos la idea de que la educación de un país (México no es la excepción), en un momento dado, es fruto de la construcción histórica, mediante procesos de mediana y larga duración en los que intervienen múltiples actores con visiones, ideologías e intereses muchas veces divergentes. Como en otros espacios de la vida social, las políticas educativas son justamente el canal a través del cual se impulsa el cambio y el ajuste (Mancebo, 2010). Por ello, el tema concita tanta discusión, diversos puntos de vista, debates en un sentido y otro; la actual reforma educativa no escapa a ese intercambio de ideas.

El nuevo paradigma en el que se sostiene la reforma es una mezcla de liberalismo económico, modernización, reducción del gasto público como resultado de la crisis fiscal, cambios en la valoración por parte de las sociedades, con exigencias de mayor participación, democratización de los procesos incluyendo los de gestión y transformaciones cada vez más aceleradas en el conocimiento científico y en las tecnologías derivadas del mismo, además de la formación de un nuevo colectivo a partir del papel de los medios (Díaz Barriga e Inclán, 2000). 
Las reformas que se implementaron en los últimos años del siglo XX se caracterizan por contar con un conjunto de metas comunes, entre las que destacan, por ejemplo, lograr mayor equidad en el acceso a la educación, mejorar la calidad y los rendimientos del servicio ofrecido, establecer un conjunto de sistemas que tiendan a informar a los usuarios (estudiantes, padres de familia y sociedad) de los resultados de la educación, revisar los contenidos que se integran al currículo y, en algunos casos, a los libros de texto. Así, se identifica una serie de problemas vinculados con la "falta de calidad de la educación", tales como altos índices de repetición, menor dominio de aprendizajes por parte de los estudiantes, falta de renovación de los métodos de enseñanza (Díaz Barriga e Inclán, 2001; Díaz Barriga, 2012), situación que perdura en la actual aplicación y propuesta de reforma educativa que vive el sistema educativo nacional.

La Reforma Integral de la Educación Básica (RIEB) establecióel compromiso de llevar a cabo una modificación curricular orientada al desarrollo de competencias y habilidades, mediante la renovación en los enfoques, asignaturas y contenidos de educación básica y la enseñanza del idioma inglés desde el nivel preescolar. Asimismo, instituyó la propuesta de profesionalizar a los maestros y a las autoridades educativas, y evaluar para mejorar; desde esta perspectiva, dicha evaluación serviría de estímulo para elevar la calidad de la educación, favorecer la transparencia y la rendición de cuentas y servir de base para el diseño adecuado de políticas educativas. En el discurso oficial la intencionalidad de la propuesta es loable, pero no señala las limitaciones de una iniciativa de esa envergadura.

La reforma educativa es parte de una política pública que impulsa (al menos en el discurso) la formación integral de todos los alumnos de preescolar, primaria y secundaria, con el objetivo de favorecer el desarrollo de competencias para la vida y el logro del perfil de egreso, a partir de aprendizajes esperados, del establecimiento de estándares curriculares, de desempeño docente y de gestión.

Para los propósitos de la actual reforma, elevar la calidad de la educación implica, necesariamente, mejorar el desempeño de todos los integrantes del sistema educativo: docentes, estudiantes, padres y madres de familia, tutores, autoridades, materiales de apoyo y el plan y los programas de estudio. Para lograrlo, es indispensable fortalecer los procesos de evaluación, transparencia y rendición de cuentas que indiquen los avances y las oportunidades de mejora, a fin de contar con una educación cada vez de mayor calidad (SEP, 2011). Como se puede constatar, la esencia de la reforma se basa en lo que ocurra al interior del aula, en el trabajo sin descanso del docente y en la posibilidad de transformar las culturas escolares, pero deja prácticamente sin responsabilidad a los actores y autoridades educativas encargadas de sostener con recursos la enorme transformación que se exige o propone a la escuela y a la educación.

En muchos países (incluido el nuestro), los maestros se topan con un doble desafío: por un lado, atender más alumnos (masificación), y por otro, atenderlos en lo que hoy se conoce como diversidad; en muchos casos estos estudiantes provienen de sectores socialmente excluidos, como consecuencia de la concentración en los sistemas de distribución de la riqueza. Así, el desfase entre el conocimiento y las competencias que pueden movilizar los docentes y los problemas que deben resolver en el salón de clases convierten a la enseñanza en un oficio que cada vez demanda mayor compromiso, que expone al individuo a un escrutinio respecto de su desempeño. A partir 
de esa condición, la inversión/inmersión personal en la tarea (trabajo concreto) puede ser gratificante, pero también puede causar situaciones de depresión, en especial cuando no se cuenta con las competencias y condiciones de trabajo adecuadas, y por lo tanto no es posible alcanzar los resultados esperados (Tenti, 2007). Dicha problemática no tendría que ser endosada de manera individual a los docentes.

Con base en lo anterior se otorga al maestro un papel de actor principal para el logro de la calidad educativa. Ahora su desarrollo profesional ya no se considera un tema periférico sino fundamental para la mejora de la escuela (Cordero et al, 2011).

Otro aspecto a considerar es la rutina de las prácticas escolares, es decir, si se cumple efectivamente la jornada, si se aprovecha el tiempo dedicado a la enseñanza, si los profesores motivan a los alumnos, si estimulan los aprendizajes, si se trabaja en un clima adecuado, si los directivos asumen su papel activo en el rumbo académico, si se involucra a los padres de familia en la tarea educativa y si se usan pertinentemente los recursos de apoyo a la enseñanza (García, 2012; Díaz Barriga, 2012). Lo anterior no debe obviar los requerimientos de infraestructura para cada una de las escuelas, modo por el cual los espacios escolares se convierten en ámbitos que verdaderamente invitan a que la comunidad escolar se sienta confortable en el quehacer de enseñar y de aprender; ello está relacionado directamente con un salario a los docentes que resignifique y dignifique la labor educativa. Sin estos elementos y sólo con base en retórica la realidad no se transforma, en todo caso se esconde, se niega, pero no se atiende.

\section{Profesionalización docente para la educación básica}

El escenario actual ha llevado a un agotamiento del rol cumplido por el profesorado en la educación tradicional, asociado principalmente a la transmisión unidireccional de información, a la memorización de contenidos, a una escasa autonomía en los diseños y evaluación curriculares, a una actitud pasiva frente al cambio e innovación educativa y a un modo de trabajar individual, más que cooperativo. Pensar y mantener el desempeño docente exclusivamente en relación con la tarea pedagógica-educativa, dentro o fuera del aula, deja al profesor en una situación inactiva respecto de la gestión y la política educativa, lo que significa mantenerlo como instrumentalizador de currículos prediseñados, como simple ejecutor de decisiones ajenas $y$, a pesar de ello, como el único responsable de los "malos" resultados de aprendizaje de sus estudiantes, situación que difícilmente cambiará mientras no se logre establecer una relación directa entre su ejercicio profesional y qué tanto se involucre en el desarrollo de la escuela y la educación.

La mayoría de los procesos de reforma se diseñaron lejos de los docentes, donde todo o casi todo está predeterminado en los manuales, guías, libros del profesor, talleres. Se insiste en la necesidad de contar con maestros innovadores que investiguen y sistematicen nuevo conocimiento, aunque hay consenso en cuanto a que se requiere un nuevo docente capaz de desenvolverse en escenarios complejos, que acceda y emplee las nuevas TIC; pero al momento de abordar su formación, su práctica y su protagonismo, se eligen las mismas vías del pasado: capacitación y más capacitación (Robalino, 2005). A lo anterior hay que agregar que los cambios recientes introducen el mecanismo de evaluación del desempeño.

De manera tradicional, la docencia se consideró como una "cuasi-profesión". En efecto, en tanto actividad comparte ciertas características típicas de las profesiones constituidas, tales como una preparación académica, la obtención de un título que habilita y garantiza una exclusividad en la realización de ciertas funciones y un conjunto de reglas éticas que conforman una deontología. Sin embargo, contrario a lo que sucede en las profesiones liberales clásicas, los docentes "trabajan en grandes organizaciones, están sometidos a un control jerárquico, no eligen a sus 
'clientes', su carrera depende inexorablemente de sus performances" (Tenti, 2007, p. 343). Esa configuración del trabajo docente significó para el profesor caer en un círculo repetitivo, basta la pura inercia de los programas y la cotidianidad de la vida en el aula; en buena medida esta dinámica se impuso desde fuera, él no decidía sobre su quehacer, todo le era dado y definido, él sólo tenía que ser docente. Ahora, y por decreto, de la noche a la mañana se pretende tener a un maestro que se transforma y transforma la cultura escolar.

\section{Referencias}

Aguilar, L. (2008). Marco para el análisis de las Políticas Públicas. Recuperado de: file:///C:/Users/ upnes/Downloads/Aguilar\%20Villanueva, $\% 20$ Luis\%20F.\%20(2008),\%20Marco\%20para\%20 an\%C3\%A1lisis\%20de\%20Pol\%C3\%ADticas\%20 P\%C3\%BAblicas.pdf

Cordero, G. et al. (2011). La profesionalización de los maestros de educación básica. Perfiles educativos, vol. XXXIII, número especial, 239-249.

Díaz Barriga, Á. e Inclán Espinosa, C. (2000). El docente en las reformas educativas. Sujeto o ejecutor de los proyectos ajenos. Buenos Aires. recuperado de: http://biblioteconomía/clacso/ gt/20101010115004/barriga.pd

Díaz Barriga, F. (2012). Reformas curriculares y cambio sistémico, una articulación ausente pero necesaria para la innovación. Revista Iberoamericana de Educación Superior, III (7), UNAM-IISUE/Universia, 23-40. Recuperado de: http://ries.universia.net/index.php/ries/article/ view/229.

Fernández, M. (2009). Educar en tiempos inciertos. Madrid: Ed. Morata.

Flores, M. del C. (2004). De la formación a la práctica docente. Un estudio de los procesos de transferencia de los profesores. Revista Latinoamericana de Estudios Educativos, vol. XXXIV, 37-68.
García, B. (2012). Consolidar las reformas de la educación básica en México. Perfiles educativos, vol. XXXIV, número especial, IISUE-UNAM, 47-56.

Mancebo, M. E. (2010). Políticas de profesionalización docente y de inclusión educativa en el Uruguay del siglo XXI: un modelo para armar. Ponencia presentada en el III Congreso Uruguayo de Ciencia Política, Montevideo, Uruguay.

Poggi, M. (2010). Prólogo. En E. Tente Fanfani (Coord.), El oficio de docente, vocación, trabajo y profesión en el siglo XXI (pp. 9-11). México: Siglo XXI editores, IIEP- UNESCO.

Popkewitz, T. S. (1995). Política, conocimiento y poder: algunas cuestiones para el estudio de las reformas educativas. Revista de Educación, núm. 305, 103-138.

Rivas, I. (2004). Política educativa y prácticas pedagógicas. Barbecho, revista de Reflexión Socioeducativa, no. 4. Recuperado de: http://www. barbecho.uma.es/DocumentosPDF/BARBECH04/A7B4. pdf

Robalino, M. (2005). ¿Actor o protagonista? Dilemas y responsabilidades sociales de la profesión docente. Revista PREAL (Proyecto Regional de Educación para América Latina y el Caribe), no. 1. Chile-UNESCO, 7-23.

SEP. (2011). Plan de estudios 2011. Educación básica. México: autor.

Tenti, E. (2007). Consideraciones sociológicas sobre profesionalización docente. Educaçäo \& Sociedade, 28(99), 335-353. Recuperado de: http://www. cedes.unicamp.br 